\title{
Autophagy promotes T-cell survival through degradation of proteins of the cell death machinery
}

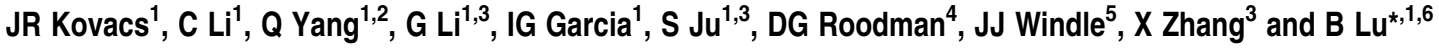

Autophagy is implicated in regulating cell death in activated T cells, but the underlying mechanism is unclear. Here, we show that inhibition of autophagy via Beclin 1 gene deletion in T cells leads to rampant apoptosis in these cells upon TCR stimulation. Beclin 1-deficient mice fail to mount autoreactive T-cell responses and are resistant to experimental autoimmune encephalomyelitis. Compared with Th17 cells, Th1 cells are much more susceptible to cell death upon Beclin 1 deletion. Cell death proteins are highly increased in Beclin 1-deficient $T$ cells and inhibition of caspases and genetic deletion of Bim reverse apoptosis. In addition, p62/sequestosome 1 binds to caspase-8 but does not control levels of procaspase-8 or other cell deathrelated proteins. These results establish a direct role of autophagy in inhibiting the programmed cell death through degradation of apoptosis proteins in activated T cells.

Cell Death and Differentiation (2012) 19, 144-152; doi:10.1038/cdd.2011.78; published online 10 June 2011

Macroautophagy (termed autophagy herein) is a cellular catabolic process that is involved in cytoplasmic renewal and turnover of long-lived proteins and cellular organelles. ${ }^{1-4}$ Autophagy is characterized by the formation of autophagosomes, which are double-membrane vesicles that sequester a portion of the cytoplasm. Yeast genetic analysis has led to the identification of more than 30 autophagy-related genes (ATG genes) involved in autophagy and its related pathways. ${ }^{5,6}$ Beclin 1 (BECN1) is the mammalian homolog of Atg6. ${ }^{7}$ BECN1 forms a complex with Vps34/class III phosphoinositide 3-kinase and is critical for multiple steps during autophagy. $^{8-11}$

Autophagy has been shown to either promote or inhibit cell death in T cells. A study showed that HIV Env protein induced autophagy and accumulation of Beclin 1 in $\mathrm{CD}^{+}$ $\mathrm{T}$ lymphocytes via CXCR4, and Env-mediated autophagy was required to trigger CD4 ${ }^{+}$T-cell apoptosis. ${ }^{12}$ Our lab has demonstrated that autophagy is highly induced in effector T cells, and blockade of autophagy using RNAi and chemical inhibitors suppresses growth factor-withdrawal-induced cell death in Th2 cells. ${ }^{13}$ More recent studies suggest that autophagy is an important death pathway in T cells lacking FADD activity, caspase-8 or Irgm-1. ${ }^{14,15}$ These findings are consistent with a role of autophagy in promoting T-cell death. In contrast, many studies suggest a pro-survival role of autophagy in $\mathrm{T}$ cells. ${ }^{16,17}$ Deletion of Atg5 resulted in decreased numbers of both thymocytes and peripheral $\mathrm{T}$ cells. ${ }^{16,17}$ In addition, more Atg5-/- $\mathrm{T}$ cells undergo apoptosis upon TCR stimulation when compared with WT T cells. ${ }^{16,17}$ Therefore, autophagy has both pro-survival and pro-death roles in $\mathrm{T}$ cells, and yet the exact molecular mechanism remains elusive.

Here, we show that Beclin 1-deficient CD4 ${ }^{+} \mathrm{T}$ cells, which lack autophagy, are prone to apoptosis upon TCR stimulation due to greatly elevated levels of latent forms of cell deathrelated proteins, such as procaspase-3, procaspase-8 and Bim. As a result, BECN1-deficient mice fail to mount autoimmune responses and are resistant to experimental autoimmune encephalomyelitis (EAE). We have further demonstrated that, compared with Th17 cells, Th1 cells are much more susceptible to cell death upon Beclin 1 deletion. Moreover, we demonstrate that caspase- 8 is present in the p62-containing protein complex and such complexes accumulate in Beclin 1-deficient T cells, suggesting a role of autophagy in mediating degradation of the procaspase-8/p62 signaling protein complex. Our study establishes a novel mechanism of apoptosis regulation in activated $\mathrm{T}$ cells via autophagy.

\section{Results}

Autophagy blockade by Beclin 1 deletion leads to a drastic increase in T-cell death upon stimulation. We have previously shown that autophagy can be induced in $\mathrm{T}$ cells. To further establish the role of autophagy in cellmediated immune responses, we studied $T$ cells from autophagy-deficient mice. BECN1 is mammalian homolog of yeast autophagy gene $\operatorname{Atg} 6 .^{8}$ It is required for both initiation and progression of autophagy. ${ }^{10,11}$ We developed CD4-cre BECN1 fl/fl (4cre BECN1 fl/fl) mice, in which BECN1

\footnotetext{
${ }^{1}$ Department of Immunology, University of Pittsburgh School of Medicine, Pittsburgh, PA, USA; ${ }^{2}$ Shenzhen - Hong Kong Institute of Infectious Disease, Shenzhen Third People's Hospital, Shenzhen, China; ${ }^{3}$ Department of Immunology, Institute of Medical Biotechnology, Soochow University, Suzhou, China; ${ }^{4}$ Department of Medicine, University of Pittsburgh School of Medicine, Pittsburgh, PA, USA; ${ }^{5}$ Department of Human and Molecular Genetics, Virginia Commonwealth University, Richmond, VA, USA and ${ }^{6}$ University of Pittsburgh Cancer Institute, Pittsburgh, PA, USA

${ }^{*}$ Corresponding author: B Lu, Department of Immunology, University of Pittsburgh School of Medicine, E1047 BSTWR, 200 Lothrop Street, Pittsburgh, PA, USA.

Tel: 412648 9339; Fax: 412383 8098; E-mail: binfeng@ @itt.edu

Keywords: T cell; Th1 cell; apoptosis; autophagy; caspase; Beclin 1

Abbreviations: EAE, experimental autoimmune encephalomyelitis; sqstm1, sequestosome 1; ATG, autophagy-related gene; TUNEL, terminal transferase dUTP nick-end labeling; MOG, myelin oligodendrocyte glycoprotein

Received 05.10.10; revised 11.4.11; accepted 02.5.11; Edited by H-U Simon; published online 10.6.11
} 
is deleted in T cells starting at the stage of $\mathrm{CD}^{+}{ }^{+} \mathrm{CD} 8^{+}$ thymocytes (Supplementary Figures 1 and 2). We observed no difference in thymic development in 4cre BECN1 fl/fl mice (Figure 1a). In spleens and lymph nodes, the number of $\mathrm{CD}^{+} \mathrm{T}$ cell was decreased more than threefold in CD4-cre BECN1 $\mathrm{fl} / \mathrm{fl}$ compared with WT (Figures $1 \mathrm{~b}$ and $\mathrm{c}$ ). In contrast, there was a modest (less than twofold) decrease in $\mathrm{CD}^{+}{ }^{+} \mathrm{T}$ cells of $4 \mathrm{cre}$ BECN1 $\mathrm{fl} / \mathrm{fl}$ mice compared with WT mice (Figures $1 b$ and $c$ ) in spleens and lymph nodes. There was no difference in percentage of naïve and memory $T$ cells between WT and 4cre BECN1 fl/fl mice (Figure 1d) in spleens. These data are in line with previous reports that autophagy blockade negatively affect peripheral T-cell homeostasis. ${ }^{16,17}$

To examine the role of autophagy in T-cell survival during T-cell clonal expansion, we stimulated CD4 ${ }^{+} \mathrm{T}$ cells from both $\mathrm{WT}$ and 4cre BECN1 fl/fl spleens for $72 \mathrm{~h}$ and quantified the percent of live cells using flow cytometry and counting (Figure $2 \mathrm{a}$ ). Compared with WT CD4 ${ }^{+}$T cells, $<10 \%$ of the activated BECN1-deficient $\mathrm{CD}^{+}{ }^{+} \mathrm{T}$ cells were alive after culture for $72 \mathrm{~h}$ (Figure $2 \mathrm{~b}$ ). For the surviving cells, however, proliferation was modestly reduced in 4cre BECN1 fl/fl CD4 ${ }^{+}$ $\mathrm{T}$ cells (Figure $2 \mathrm{a}$, right panel). This result suggests that activation of Beclin 1-deficient T cells with anti-CD3 and antiCD28 results in massive cell death. Consistent with apoptosis in mediating cell death, many $4 \mathrm{cre}$ BECN1 fl/fl CD4 ${ }^{+} \mathrm{T}$ cells exhibited condensed nuclei with apoptotic chromatin (Figure 2c). In addition, there was a clear increase in terminal transferase dUTP nick-end labeling (TUNEL) signals for 4cre BECN1 fl/fl CD4 ${ }^{+}$T cells when compared when WT CD4 ${ }^{+}$ $T$ cells (Figure $2 \mathrm{~d}$ ). These data indicate that activated $4 \mathrm{cre}$ BECN1 fl/fl CD4 ${ }^{+} \mathrm{T}$ cells may die by the apoptotic death pathway. In contrast to peripheral CD4 ${ }^{+} \mathrm{T}$ cells, we observed no difference in TUNEL staining between WT and 4cre BECN1 fl/fl thymocytes after stimulation with anti-CD3 and anti-CD28 (Figure 2e). This indicates further that the increase in apoptotic cell death is specific to peripheral Beclin 1-deficient $T$ cells upon TCR stimulation. Previous reports suggest that Atg5 and Atg7, through autophagy, regulate the number of mitochondria in T cells. ${ }^{17,18}$ These studies showed that lack of Atg5 or Atg7 in the double-negative thymocytes led to an increase in the number of mitochondria and made T cells a
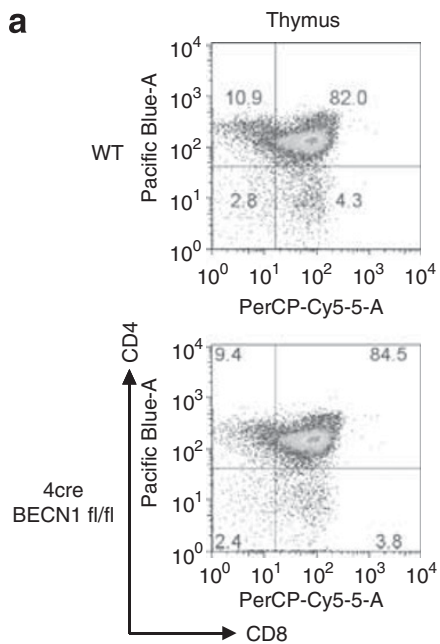

C
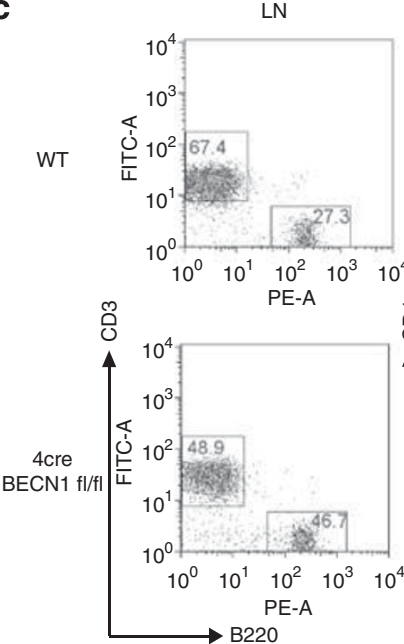

b

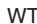

4 cre BECN1 fl/fl
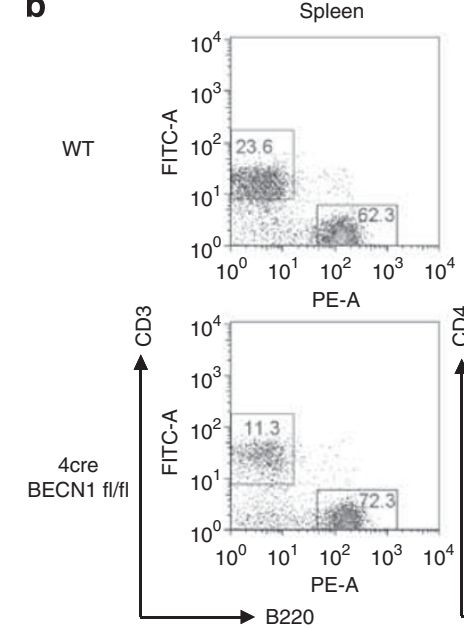
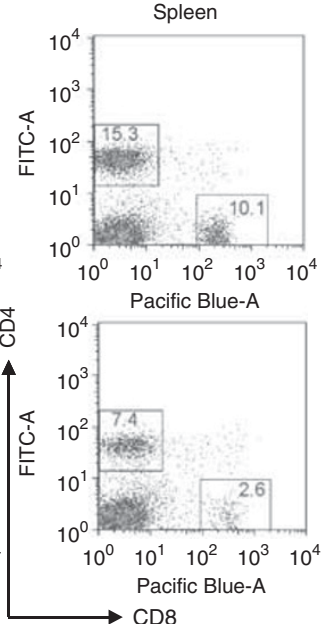

d
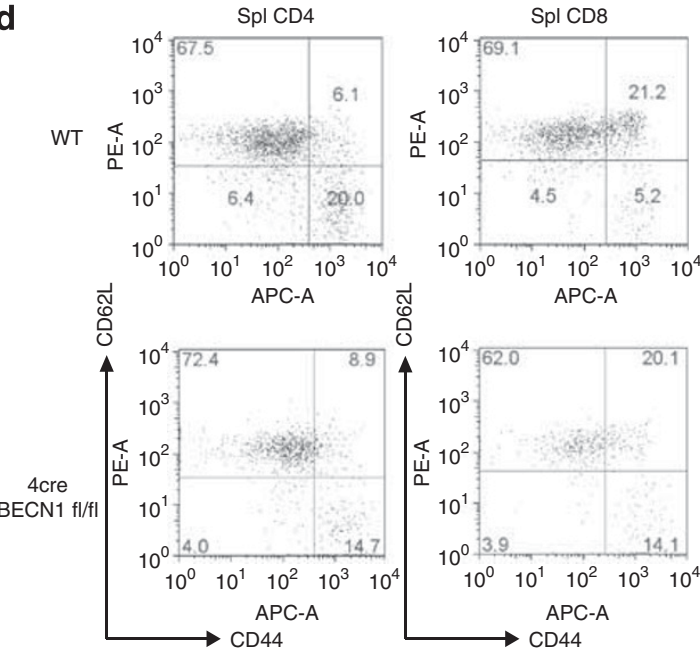

Figure 1 Modest reduction of peripheral $\mathrm{CD} 4^{+} \mathrm{T}$ cells in 4cre BECN1 fl/fl mice. Flow cytometry analysis of single-cell suspensions from thymus (a), spleen (b) and lymph node (c), which were stained for surface markers CD4, CD8, CD3 and B220. (d) Analysis of naïve and memory T cells (CD4, left; CD8, right), which were stained for CD44 and CD62L 
a

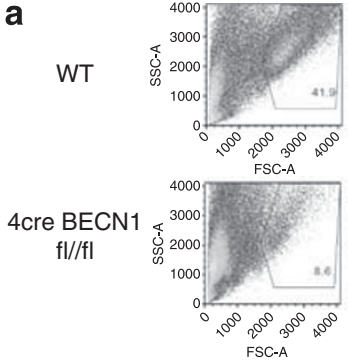

d

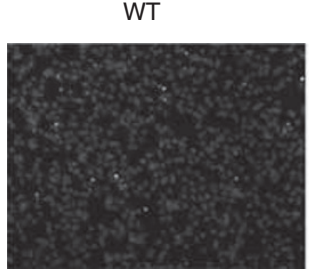

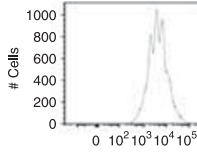

Comp-FITC-A

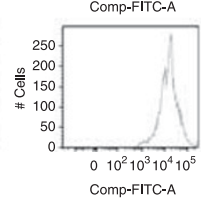

Comp-FITC-A b

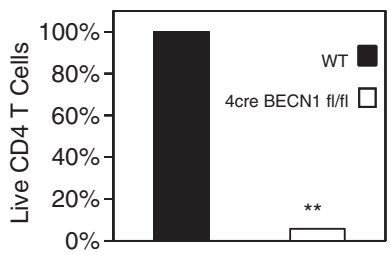

e

4cre BECN1 fl/f

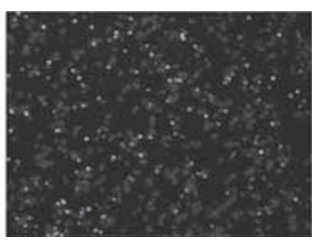

WT DP Thymus

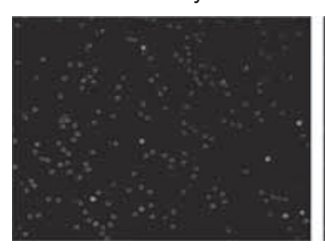

4cre BECN1 fl/fl
C

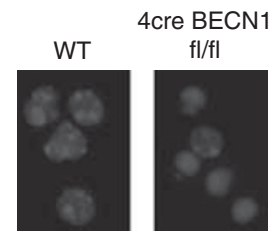

4cre BECN1 fl/fl

DP Thymus

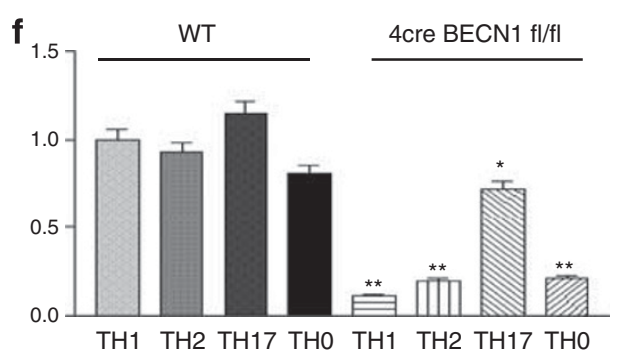

Figure 2 Beclin 1-deficient CD4 ${ }^{+} T$ cells undergo increased levels of apoptotic cell death upon TCR stimulation. CD4 ${ }^{+}$T cells were isolated from spleens of WT (top) and 4cre BECN1 fl/fl mice (bottom), stained with CFSE and stimulated under Th1 conditions for $72 \mathrm{~h}$. Cell death was assessed by flow cytometry by forward and side scatter gating of blasted lymphocytes (a). The survival of WT cells was set as 100\%, and the percentage of live Beclin 1-deficient CD4 ${ }^{+}$T cells versus WT cells were quantified (b). The morphology of cell nuclei was revealed by fluorescent microscopy after staining cells with Hoechst 33342 dye (blue; c). (d) WT and 4cre BECN1 fl/fl CD4 ${ }^{+}$T cells were stimulated by anti-CD3 and anti-CD28 for $24 \mathrm{~h}$. TUNEL (green) assay was performed and counter stained by Hoechst 33342 dye (blue). (e) TUNEL analysis was performed for double-positive (DP) and single-positive (CD4 ${ }^{+}$, SP) thymocytes upon anti-CD3 and anti-CD28 stimulation for $24 \mathrm{~h}$. All results are representative of $n \geq 3$ independent experiments. (f) Naïve CD4 ${ }^{+}$T cells were isolated from spleens of WT and 4cre BECN1 fl/fl mice and cultured under Th1, Th2, Th17 and Th0 conditions for $72 \mathrm{~h}$. Cell death was assessed by flow cytometry by forward and side scatter gating of blasted lymphocytes. The percentage of live WT cells in the Th1 condition was set as $100 \%$. Ratio of surviving Beclin 1-deficient CD4 ${ }^{+} \mathrm{T}$ cells cultured in other conditions versus the Th1 condition was calculated and shown. Student's $t$-test was performed to compare WT with Beclin 1-deficient $\mathrm{CD}^{+} \mathrm{T}$ cells cultured in the same conditions. ${ }^{*} P<0.05$ and ${ }^{* *} P<0.001$. The color reproduction of this figure is available at the Cell Death and Differentiation Journal online

more susceptible to cell death. As BECN1 is crucial for autophagy, it is possible that BECN1 regulates the number of mitochondria, and thus BECN1 deletion might lead to an increase in the number of mitochondria, thereby sensitizing cells to mitochondrion-mediated cell death. We analyzed mitochondrial accumulation by MitoTracker Green (Invitrogen, Carlsbad, CA, USA) staining of peripheral blood lymphocytes and could not find significant difference in MitoTracker Green staining between WT and 4cre BECN1 $\mathrm{fl} / \mathrm{fl} \mathrm{T}$ cell in either $\mathrm{CD}^{+}$or $\mathrm{CD}^{+}$populations (Supplementary Figures $3 \mathrm{~A}$ and $B$ ). This result suggests that Beclin 1 is not required for controlling the number of mitochondria in peripheral $\mathrm{T}$ cells.

We have previously shown that the number of autophagosomes is higher in CD4 ${ }^{+}$T cells cultured in the Th2 condition than those cultured in the Th1 condition, ${ }^{13}$ suggesting that autophagy is differentially regulated in different $\mathrm{T}$ helper cell subsets. We then decided to determine whether CD4 ${ }^{+}$T cells cultured in various polarizing conditions were differentially susceptible to cell death upon Beclin 1 deletion. We cultured naïve WT and 4cre BECN1 fl/fl CD4 ${ }^{+}$T cells in Th0, Th1, Th2 and Th17 polarizing conditions for $72 \mathrm{~h}$ and subsequently quantified the percent of live cells using flow cytometry and counting. The $4 \mathrm{cre}$ BECN1 fl/fl CD4 ${ }^{+}$T cells cultured in Th0 (about $20 \%$ of WT levels), Th1 (about $10 \%$ of WT levels) and Th2 (about $20 \%$ of WT levels) conditions were much more susceptible to cell death than WT CD4 ${ }^{+}$T cells (Figure 2f). In contrast, 4 cre BECN1 fl/fl CD4 ${ }^{+}$T cells cultured in the Th17 condition were relatively less susceptible to cell death. The number of live Beclin 1-deficient T cells was about $60 \%$ of WT $\mathrm{CD}^{+}{ }^{+} \mathrm{T}$ cells cultured in the Th17 condition (Figure 2f). These data suggest that different $T$ helper cell subsets are differently susceptible to cell death upon autophagy blockade.

Autophagy blockade leads to resistance of EAE induction. As BECN1-deficient $\mathrm{CD}^{+} \mathrm{T}$ cells undergo drastic apoptosis upon activation, we postulate that BECN1 deletion inhibits autoimmune responses. We first examined the ability of $4 \mathrm{cre}$ BECN1 $\mathrm{fl} / \mathrm{fl}$ mice to develop a T-cellmediated autoimmune disease EAE. The 4cre BECN1 fl/fl 
a

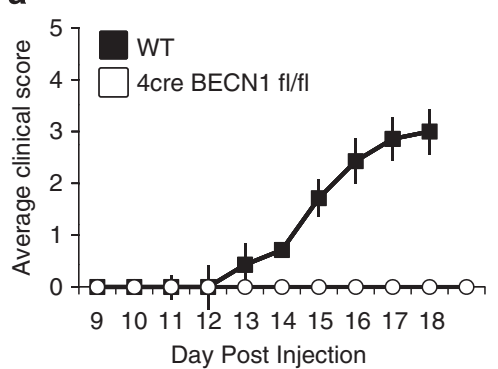

b

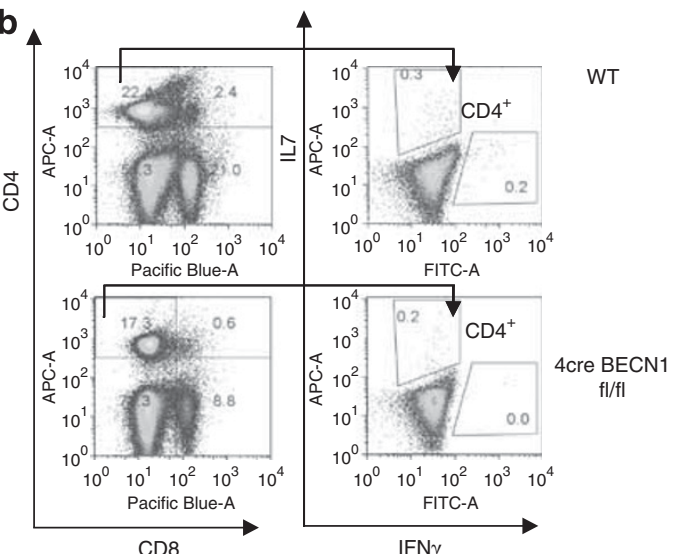

C

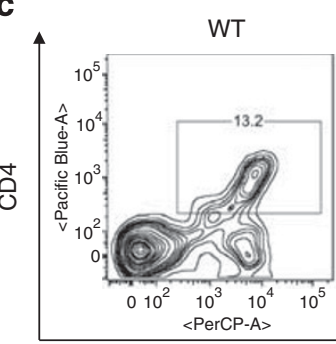

4cre BECN1 fl/fl

d

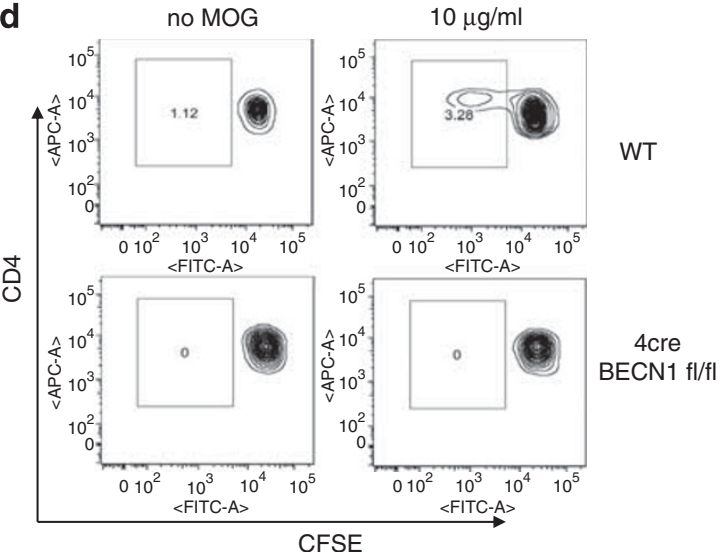

Figure 3 The clinical course of active EAE in 4cre BECN1 fl/fl mice is inhibited. (a) Active EAE was induced in WT (filled circles, $n=14$ ) and Beclin 1-deficient mice (open circles, $n=6$ ). Clinical disease was monitored for 20 days as described in the Materials and Methods. (b) Splenocytes from WT or Beclin 1-deficient mice induced for EAE (day 15) were cultured with MOG peptide $(20 \mu \mathrm{g} / \mathrm{ml})$. After 18-h culture, the cells were harvested and intracellular staining was performed (IL-17 and IFN- $\gamma)$, and cytokineproducing $\mathrm{CD}^{+}{ }^{+}$T cells were analyzed by flow cytometry. (c) CNS-infiltrating lymphocytes were isolated from WT or Beclin 1-deficient mice induced for EAE (day 15) and analyzed by flow cytometry (CD4 and CD45). (d) Splenocytes were isolated from mice that had been induced for EAE for day 15. These cells were stained with CFSE, cultured with the MOG peptide $(20 \mu \mathrm{g} / \mathrm{ml})$ for $72 \mathrm{~h}$ and subsequently analyzed by flow cytometry to determine an antigen-specific proliferating population of CD4 ${ }^{+}$T cells

mice showed complete resistance to EAE, whereas in the same experiment, all WT control mice developed severe signs of EAE (Figure 3a). To determine the mechanisms by which $4 \mathrm{cre}$ BECN1 fl/fl mice are resistant to EAE induction, we first examined ex vivo recall proliferation using mice immunized with myelin oligodendrocyte glycoprotein (MOG) peptide 10 days previously. We have found a very minor decrease in the total CD4 ${ }^{+}$T-cell number in spleens and lymph nodes in 4cre BECN1 $\mathrm{fl} / \mathrm{fl}$ after immunization (Figure 3b. left panel). In contrast, MOG-reactive IFN- $\gamma^{+}$ $\mathrm{CD}^{+}{ }^{+} \mathrm{T}$ cells were greatly reduced in 4cre BECN1 $\mathrm{fl} / \mathrm{fl}$ mice (Figure $3 \mathrm{~b}$ ). ${\mathrm{IL}-17^{+}}^{+} \mathrm{CD}^{+} \mathrm{T}$ cells were also modestly reduced (Figure $3 b$ ). These data are consistent with our in vitro data that showed that Th17 cells were less susceptible to cell death than Th1 cells upon autophagy blockade. Additionally, CD4 ${ }^{+} \mathrm{T}$ cells infiltrating the CNS were examined by flow cytometry at the peak of the clinical disease (15-20 days). CNS-infiltrating T cells were evident in WT mice but not detected in 4cre BECN1 $\mathrm{fl} / \mathrm{fl}$ mice (Figure $3 \mathrm{c}$ ). Finally, the proliferation of $\mathrm{MOG}_{35-55}$ peptidespecific T-cell populations was analyzed using CFSE assay. After 72-h culture with $\mathrm{MOG}_{35-55}$, only WT cells showed a clear proliferating population with diluted CFSE, whereas splenic cells of immunized 4cre BECN1 fl/fl mice showed only a minimal population of proliferating cells comparable to that of those cells unexposed to $\mathrm{MOG}_{35-55}$ (Figure 3d). Thus, Beclin 1 is critical for autoimmune responses.
Autophagy blockade leads to an increase in cell death-related proteins. To study further how autophagy regulates cell survival, we decided to examine whether autophagy is involved in regulating cell death-related proteins. We stimulated CD4 ${ }^{+} \mathrm{T}$ cells from WT and 4cre BECN1 fl/fl mice for $48 \mathrm{~h}$ and examined levels of cell deathrelated proteins by western blot. p62/sequestosome 1 (sqstm1) is a protein that is selectively degraded by autophagy and can be used as a readout for autophagy flux. ${ }^{19,20}$ Strikingly, the protein level of p62 is greatly increased in BECN1-deficient $T$ cells when compared with WT, suggesting autophagy is inhibited in BECN1-deficient $T$ cells (Figure 4a). Interestingly, p62 was not expressed in naïve $\mathrm{CD}^{+}{ }^{+} \mathrm{T}$ cells or $\mathrm{T}$ cells stimulated for $24 \mathrm{~h}$ (Supplementary Figure 4), suggesting the accumulation of p62 depended on prolonged T-cell activation. In contrast, p53, a substrate for ubiquitin-proteasome degradation pathway is expressed at equal levels between WT and BECN1-deficient CD4 ${ }^{+}$T cells (Supplementary Figure 4). These data are consistent with a functional deficiency in autophagy rather than the ubiquitinproteasome pathway in BECN1-deficient $\mathrm{CD}^{+}{ }^{+} \mathrm{T}$ cells. In addition, we have found a drastic increase in protein levels, but not mRNA levels, for many pro-death members of apoptotic machineries, such as caspase-8, caspase-3 and Bim (Figure 4b, Supplementary Figure 5), in Beclin 1-deficient $\mathrm{CD}^{+}{ }^{+} \mathrm{T}$ cells. These data suggest that autophagy is required for degradation of these cell death-related proteins. Bcl-2, a 
a

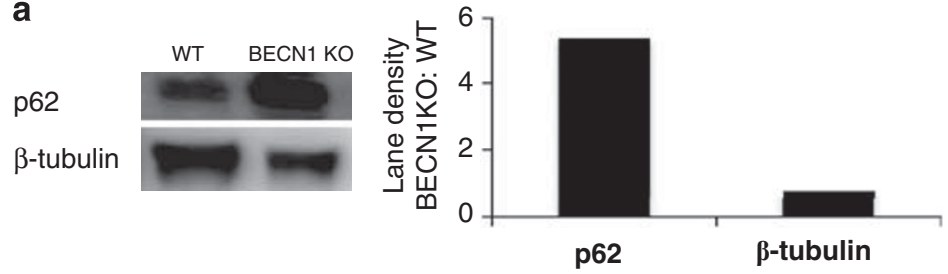

b

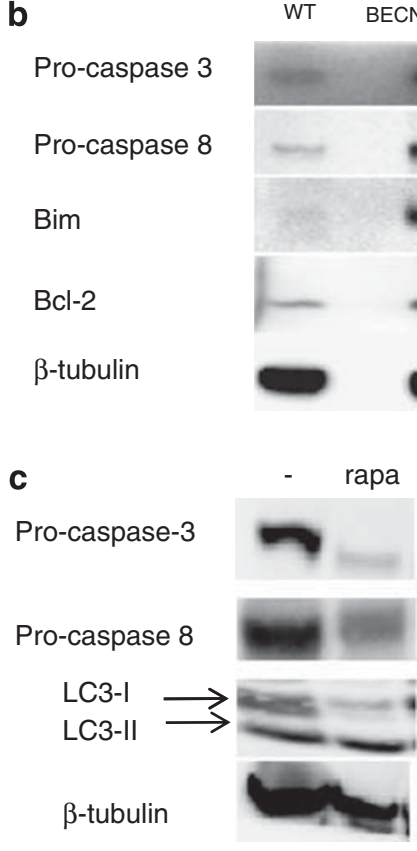

BECN1 KO

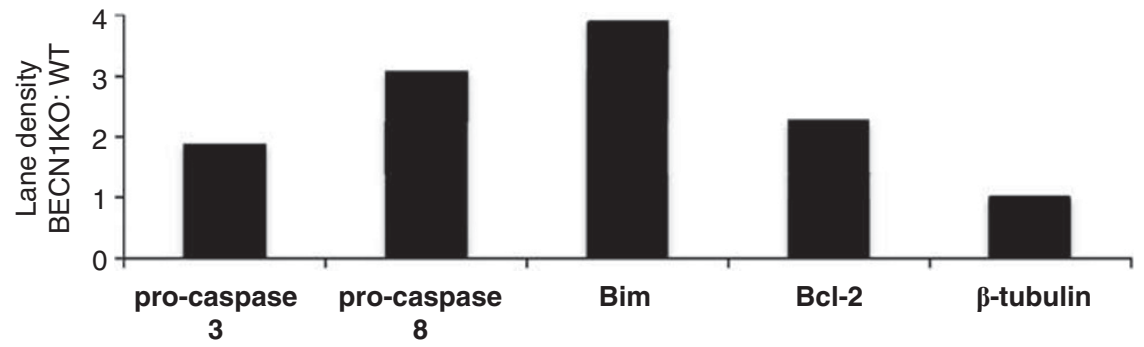

d

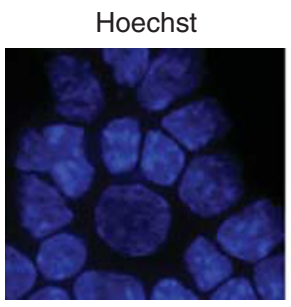

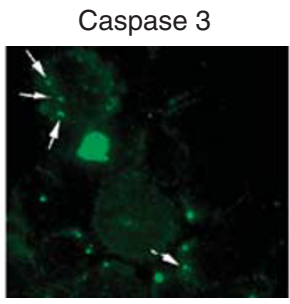

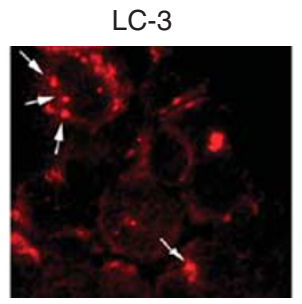

Caspase3/ LC-3/Hoechst

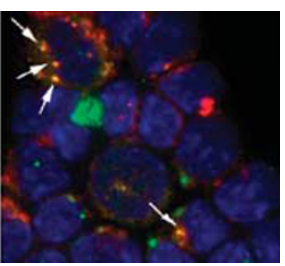

Figure 4 Beclin 1-deficient CD4 ${ }^{+} T$ cells accumulate cell death-related protein upon activation. WT and 4cre BECN1 fl/fl CD4 ${ }^{+} \mathrm{T}$ cells were stimulated for $48 \mathrm{~h}$ by anti$\mathrm{CD} 3$ and anti-CD28. At this point, $\mathrm{CD} 4^{+}$cells were re-isolated by positive selection and extract was made for western analysis. The results shown are western blot analyses of p62 (a) and apoptosis proteins, caspase-3, Bim, Bcl-2 and caspase-8 (b). (c) Rapamycin was added at $44 \mathrm{~h}$, and extracts were made at $48 \mathrm{~h}$ subjected to western blot analysis. (d) Naïve WT CD4 ${ }^{+}$T cells were cultured in the Th1 condition for $72 \mathrm{~h}$ and then treated with rapamycin for $4 \mathrm{~h}$. Cells were stained with antibodies as indicated. Fluorescence images were obtained. Arrows indicate dots that are positive for both caspase-3 and LC-3

pro-survival protein, was also modestly upregulated in BECN1-deficient $T$ cells (Figure 4b). However, its function was likely compromised due to the high levels of Bim and caspases. Therefore, procaspase- 3 and other cell deathrelated proteins seem to be degraded via the autophagylysosome pathway. Consistent with this idea, when cells were cultured in the presence of rapamycin, which induces autophagy, the protein levels of caspase- 3 and -8 were diminished (Figure 4c). In addition, we have found colocalization of LC-3 and caspase- 3 in T cells treated with rapamycin (Figure $4 \mathrm{~d}$ ). These data suggest that autophagy blockade might sensitize $\mathrm{CD}^{+}{ }^{+} \mathrm{T}$ cells to both caspasedependent and caspase-independent programmed cell death pathways. $^{21}$

Bim and caspases mediate cell death upon autophagy blockade in activated CD4 $^{+} \mathbf{T}$ cells. We further established the functional significance of caspases and Bim upregulation in cell death caused by autophagy inhibition. Addition of a pan-caspase inhibitor (zVAD) but not a Rip1 inhibitor (necrostatin 1) ${ }^{21-23}$ was able to partially reverse cell death in Beclin 1-deficient $T$ cells (Figure 5b), suggesting caspase-dependent apoptosis but not programmed necroptosis was involved in mediating the deleterious effect of autophagy inhibition. As caspase-independent cell death is mainly mediated by mitochondrion-mediated cell death pathway ${ }^{21}$ in which Bim has a critical role, ${ }^{24-28}$ we further studied the role of Bim in cell death by autophagy inhibition using $T$ cells from BECN1/Bim double-deficient mice. Numbers of both $\mathrm{CD}^{+}$and $\mathrm{CD}^{+}{ }^{+} \mathrm{T}$ cells were elevated in BECN1/Bim double-deficient mice compared with BECN1-deficient mice (Figure 5a). BECN1/Bim double-deficient $\mathrm{CD}^{+}{ }^{+} \mathrm{T}$ cells were much more resistant to cell death upon TCR stimulation compared with BECN1deficient mice, although they were still more susceptible to cell death compared with WT CD4 ${ }^{+}$T cells (Figure $5 \mathrm{~b}$ ). Blockade of caspases using zVAD further protected BECN1/ Bim double-deficient $\mathrm{CD}^{+}{ }^{+} \mathrm{T}$ cells from cell death and increased the level of surviving BECN1/Bim double-deficient $\mathrm{CD}^{+}{ }^{+} \mathrm{T}$ cells to above $60 \%$ of the level of WT CD4 ${ }^{+} \mathrm{T}$ cells (Figure 5b). Collectively, these data support the idea that autophagy increases cell survival by degrading cell deathrelated proteins.

Caspase-8 is found in protein aggregates containing p62 and ubiquitin. Full apoptosis commitment necessitates 
a

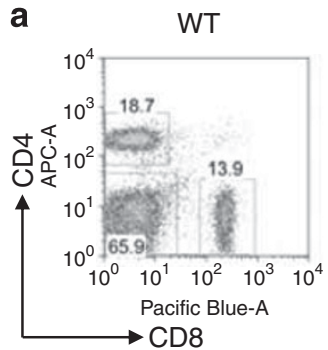

b
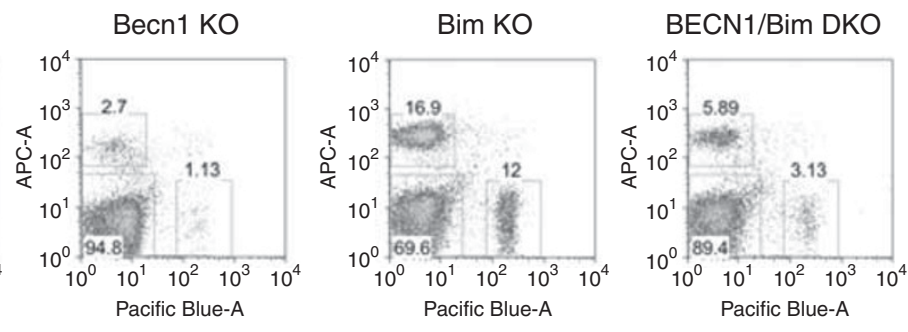

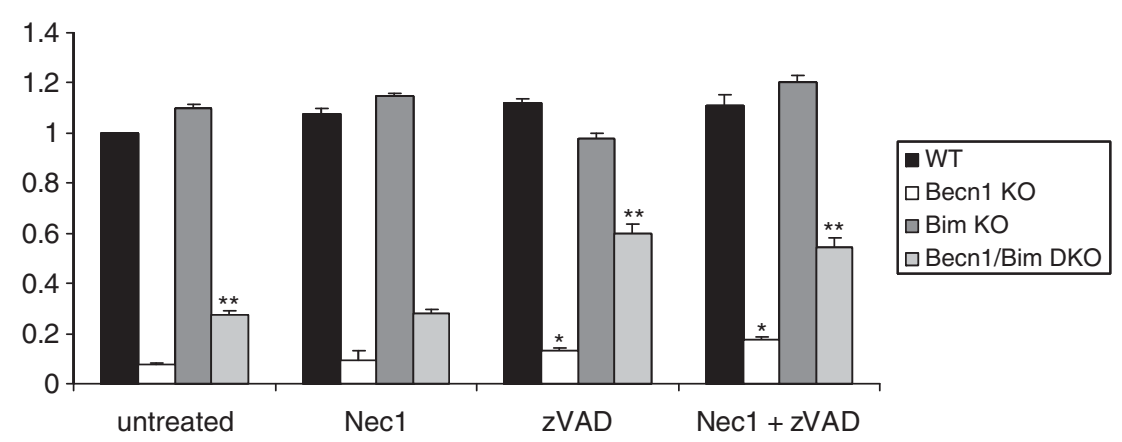

Figure 5 Caspases and Bim are involved in cell death upon autophagy blockade. (a) Flow cytometry analysis of splenocytes from WT, 4cre BECN1-/-, Bim-I- and 4 cre BECN1-I- Bim-l- mice. (b) CD4 ${ }^{+}$T cells from WT, 4cre BECN1 fl/fl, Bim $-/-$and BECN1/BIM DKO were stimulated for $72 \mathrm{~h}$ with or without chemical inhibitors. Results are presented as a survival ratio based on live cells relative to that of untreated WT cells, which is set as 1 . Pairwise $t$-test statistical analysis was completed for each treatment group relative to untreated BECN1 fl/fl group survival ratio. ${ }^{* *} P<0.001$ and ${ }^{*} P<0.05$

aggregation of initiator caspases such as casapse-8. ${ }^{29} \mathrm{~A}$ recent study shows that after proapoptotic stimulation by death receptors, caspase-8 undergoes CUL3/RBX1dependent polyubiquitination, which promotes p62mediated aggregation and full activation of the protease. ${ }^{30}$ Other studies show that clearing of ubiquitinated protein aggregates by autophagy can be facilitated by p62, which contains an ubiquitin-binding domain and an LC3-interacting region, and serves as a bridge between autophagosomes and ubiquitinated proteins that are present in protein aggregates. $^{20,31}$ On the basis of these findings, it is possible that caspase- 8 is selectively degraded via the autophagosome-lysosome pathway after being recruited by p62-containig complex. In WT $\mathrm{CD}^{+}{ }^{+} \mathrm{T}$ cells, caspase-8 colocalized with p62 in small dots, which were likely small protein aggregates (Figure 6a). In contrast, caspase-8 colocalized with p62 in much larger protein complexes in Beclin 1-deficient CD4 ${ }^{+}$T cells (Figure 6a). Recruitment of caspase- 8 into these protein complexes might be achieved via ubiquitin and p62. Indeed, a lot of these protein aggregates were also positive for ubiquitin (Figure 6a, very right panel). Consistent with these data, caspase-8 was also found to be co-immunoprecipitated with p62 (Figure 6b). Caspase-3 and Bim, however, could not be detected in these immunoprecipitates (data not shown). To determine whether p62 is required for caspase-8 degradation, we measured levels of procaspase- 8 protein in WT and p62-deficient Th1 cells generated in culture. We found that levels of procaspase-8 protein were similar between WT and p62deficient $\mathrm{CD}^{+}{ }^{+} \mathrm{T}$ cells (Figure $6 \mathrm{c}$ ). Collectively, these data suggest that the main role of $p 62$ is likely to promote caspase activation rather than to recruit caspases to autophagymediated degradation.

\section{Discussion}

Autophagy has been implicated in both cell survival and cell death in T cells. To start to resolve these contradictory results, a better understanding of the molecular targets of autophagymediated cellular events is much needed. In this study, we have found that autophagy is critical for degradation of the cell death-related proteins in CD4 ${ }^{+}$T cells, providing a molecular mechanism for pro-survival function of autophagy in activated $\mathrm{T}$ cells In contrast to the ubiquitin-proteasomal pathway, which is well known for mediating the degradation of active forms of cell death protein such as cleaved caspase- $3,{ }^{32}$ autophagy is now implicated in the degradation of the latent form of cell death protein such as procaspase-3 and -8. Downregulating autophagy in Th1 cells is likely an important mechanism, which makes Th1 cells more susceptible to apoptosis. ${ }^{33}$ Our results support the idea that regulation of latent cell death protein turnover is a critical self-limiting mechanism for activated T cells. Autophagy inhibition might be a promising therapeutic approach for the treatment of autoimmune diseases such as multiple sclerosis.

Formation of protein complexes is a critical step for signal transduction. Death receptor signaling triggers formation of a death-inducing signaling complex, ${ }^{34}$ leading to initial caspase8 activation by dimerization. However, full apoptosis commitment requires more massive stimulation, likely achieved through further aggregation of caspase- $8 .{ }^{30}$ A recent study showed that caspase-8 undergoes polyubiquitination and is present in high-molecular-weight protein complexes after death receptor stimulation. ${ }^{30}$ Such high-molecular-weight protein complexes serve as amplifiers of death signals and are likely crucial for apoptosis. We have found in our study that autophagy blockade leads to accumulation of protein 
a

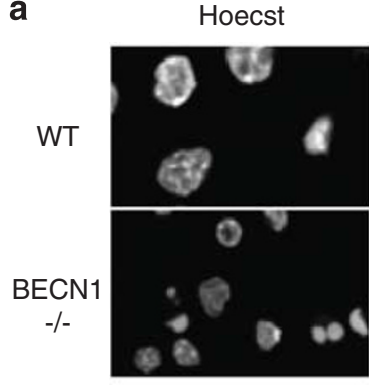

p62/SQSTM1

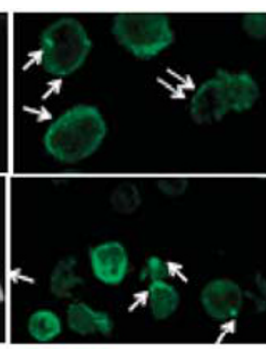

Caspase 8

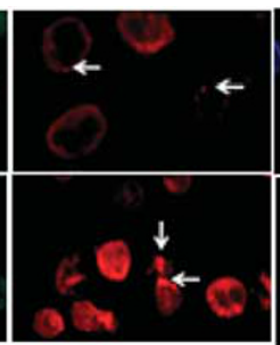

Ubiquitin

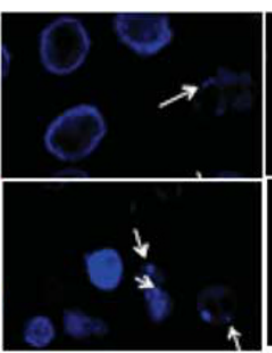

p62/Casp8

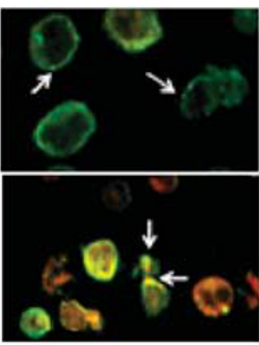

p62/Casp8/Ub

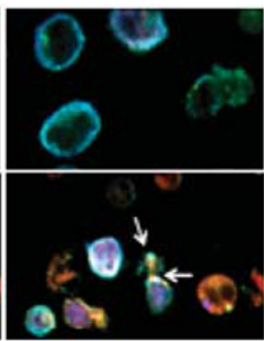

b

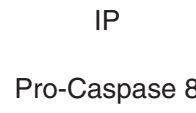

p62
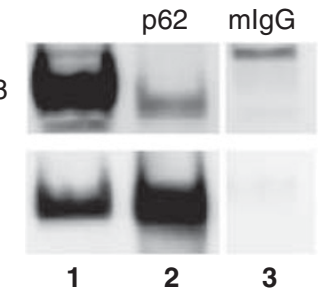

c

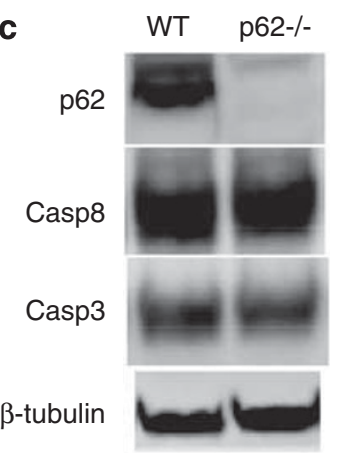

Figure 6 Caspase-8 is found in p62/ubiquitin-containing aggregates in BECN1-deficient T cells. (a) WT and 4cre BECN1 fl/fl CD4 + T cells were stimulated for $48 \mathrm{~h}$ by anti-CD3/anti-CD28 (arrows pointing to aggregates). Cells were stained with antibodies, as indicated. Confocal images were obtained. (b) CD4 ${ }^{+}$T-cell lysates were immunoprecipitated with anti-p62 antibodies (lane 2) and subjected to western blot with antibodies, as indicated. Extracts equal to 1/20 of the amount used for immunoprecipitation was used as control (lane 1). (c) Naïve WT and p62-/- CD4 ${ }^{+}$T cells were stimulated for $48 \mathrm{~h}$ by anti-CD3 and anti-CD28. Extracts were made for western blot analysis for p62, caspase-3, caspase-8 and $\beta$-tubulin, as indicated. The results are representative of three independent experiments. One pair of mice was used for each experiment

complexes containing caspase-8, correlating with massive apoptosis in these cells. These data reveal a novel mechanism of downregulation of death receptor signaling via autophagy. It is possible that autophagy also inhibits signaling in other signal transduction pathways via selective degradation of certain signaling complexes. How other cell deathrelated proteins degraded by autophagy remains to be studied further.

Two recent reports showed that deletion of Atg5 and Atg7 during T-cell development resulted in an increase in the number of mitochondria in both thymocytes and peripheral T cells. ${ }^{17,18}$ However, our study could not confirm these results. One possible reason could be because of the fact that Lck-cre was used in the previously published work, resulting in cre expression at the double-negative stage during thymic T-cell development. In contrast, we used CD4-cre transgenic mice. In our case, cre is expressed at double-positive stage during thymic T-cell development. As autophagy is turned off later during $\mathrm{T}$-cell development in our mice and we did not see accumulation of more mitochondria in Beclin 1-deficient $T$ cells, it is possible that autophagy controls the number of mitochondria at an early stage of T-cell development in thymus. Indeed, the total thymocytes are similar between WT and $4 \mathrm{cre} \mathrm{BECN} 1 \mathrm{fl} / \mathrm{fl}$ mice. In contrast, total thymocytes were decreased in conditional Atg5-deficient mice and conditional Atg7-deficient mice when compared with WT controls. ${ }^{17,18}$ Consistent with this idea, a recent study showed that it took 18 days for mitochondria to accumulate in cultured T cells. ${ }^{35}$ It is also possible that, although the total number of mitochondria is not changed, there is a deficiency in clearance of damaged mitochondria in Beclin 1-deficient T cells. We have examined this possibility via quantifying levels of reactive oxygen species (ROS) within $\mathrm{T}$ cells by staining $\mathrm{CD}^{+}{ }^{+} \mathrm{T}$ cells cultured in the Th1 condition with $2^{\prime}-7^{\prime}$-dichlorodihydrofluorescein diacetate. Using this method, we have found no difference in the levels of ROS in WT and BECN1-deficient T cells (Supplementary Figure 6). In addition, we also inhibited ROS by adding cell-permeable superoxide dismutase mimetic MnTBAP and antioxidant Tiron to cell culture. We found these chemicals were not able to rescue cell death in BECN1deficient $T$ cells (data not shown). These data are consistent with lack of a significant role of Beclin 1 in mitochondrial clearance during early T-cell activation. Another possible reason could be due to the fact that Beclin 1, Atg5 and Atg7 are differentially required for mitophagy (autophagic degradation of mitochondria) in $T$ cells. Regardless of these possibilities, in $T$ cells, the increase in the sensitivity to apoptosis in the absence of autophagy does not appear to be due to a change in the number of mitochondria.

In this study, we have shown that Beclin 1 is critical for TCRinduced expansion of T cells. The lack of Beclin 1 resulted in massive T-cell death upon TCR stimulation. In addition, Beclin 1 deletion in $T$ cells resulted in failure to mount cell-mediated autoimmune responses. The pro-survival function of Beclin 1 is, however, not seen when T cells are cultured in the absence of TCR stimulation or in thymocytes stimulated with anti-CD3 (our unpublished observation). Our previous publication showed that autophagy actually promotes cell death during cytokine-withdrawal cell death in a Th2 cell line. ${ }^{13}$ Whether autophagy is pro-survival or pro-death might be highly 
dependent on the cellular context and stimuli. Interestingly, we have also found that $\mathrm{CD} 4^{+} \mathrm{T}$ cells cultured in different polarizing conditions were differentially susceptible to cell death. Beclin 1-deficient CD4 ${ }^{+} \mathrm{T}$ cells cultured in the Th1 condition were most susceptible to apoptosis, whereas same $\mathrm{T}$ cells cultured in the Th17 condition were much less susceptible to apoptosis. These data are consistent with our in vivo finding in the EAE model, in which MOG-specific Th17 responses were only modestly affected in Beclin 1-deficient mice, whereas Th1 responses were severely compromised. These data suggest that autophagy is differentially required for the survival of T-cell subsets. Blockade of autophagy will likely change the balance of different T-cell subsets during immune responses.

During the revision of this paper, a report showed lack of a role of Beclin 1 in peripheral T cells. ${ }^{36}$ They propose that Beclin 1-independent autophagy is operational in Beclin 1-deficient $T$ cells. Our work, however, demonstrates that Beclin 1 is required for autophagy in T cells. The discrepancy in our findings could be attributable to differences in mouse genetic backgrounds. It is also possible that the Beclin 1 'knockout' strategy in that study did not result in a total ablation of Beclin 1 protein.

Despite the fact that we have tried to inhibit both mitochondrion- and caspase-mediated programmed cell death pathways, there are still significantly higher levels of cell death in $\mathrm{CD}^{+}{ }^{+}$T cells lacking autophagy. It is known that autophagy is required to provide mitochondria with substrates to maintain oxidative phosphorylation during nutrient deprivation. A cellpermeable form of pyruvate, methyl pyruvate (MP), was shown to reverse autophagy blockade-induced cell death in Bax-/Bak-/- cells. ${ }^{37}$ We also added MP to BECN1-/- CD4 ${ }^{+} \mathrm{T}$ cells and found no evidence that MP can rescue cell death in these cells (data not shown). Additional mechanisms might be operative in causing cell death upon autophagy blockade.

\section{Materials and Methods \\ Generation of Beclin 1 conditional-deficient mice. The 10.4-kb genomic fragment containing Beclin-1 exons 1-3 was subcloned from the bacterial artificial chromosome clone (RPC123-145H19) obtained from PJde Jong (Children's Hospital, Oakland, CA, USA). Using homologous recombination techniques in bacteria, we inserted a loxp site (filled arrow) in front of exon 1 and another loxp site downstream of exon 2. The vector was electroporated into SW9.5 ES cells, a gift from $R$ Chaillet (University of Pittsburgh). Drug-resistant mouse embryonic stem cell clones were screened by Southern blot. Two independent correctly targeted embryonic stem cell clones were injected into C57BL/6 blastocysts, and germline transmission was obtained from each clone. The Neo gene was removed by breeding $\mathrm{F} 1$ mice with a strain of actin promoter-driven Flippase transgenic mice (The Jackson Laboratory, Bar Harbor, MA, USA). These mice were then bred with $\mathrm{CD} 4$-cre to generate $\mathrm{CD} 4-\mathrm{cre} \mathrm{BECN} 1 \mathrm{fl} /{ }^{+}$. These mice were intercrossed to generate CD4-cre BECN1 fl/fl mice. All animals were maintained under specific pathogen-free conditions. The Institution Animal Care and Use Committee at University of Pittsburgh have approved all animal work.}

Cell culture. Mouse naïve $\mathrm{CD} 4^{+} \mathrm{T}$ cells $\left(\mathrm{CD} 44^{-} \mathrm{CD} 62 \mathrm{~L}^{+}\right)$were purified from spleens and lymph nodes of C57BL/6 mice. These cells were then cultured on 24-well plates precoated with $10 \mu \mathrm{g} / \mathrm{ml}$ plate-bound anti-CD3 (clone 145-2C11) and $5 \mu \mathrm{g} / \mathrm{ml}$ plate-bound anti-CD28 mAbs (clone 37.51) in complete RPMI (RPMI 1640 supplemented with $10 \%$ heat-inactivated FCS, $2 \mathrm{mM}$ L-glutamine, $50 \mu \mathrm{M}$ 2-ME, $100 \mathrm{U} / \mathrm{ml}$ penicillin and $100 \mu \mathrm{g} / \mathrm{ml}$ streptomycin) in various polarizing conditions: Th1, hulL-2 $(20 \mathrm{U} / \mathrm{ml}$, obtained from the BRB Preclinical Repository), IL-12 $(3.4 \mathrm{ng} / \mathrm{ml})$ plus anti-IL-4 $(10 \mu \mathrm{g} / \mathrm{ml}$, clone $11 \mathrm{~B} 11$, from the BRB Preclinical Repository); Th0, hulL-2 (20 U/ml) for the Tc0 condition; Th2, hulL-2 (20 U/ml), IL-4
$(2 \mathrm{ng} / \mathrm{ml})$ and anti-IFN- $\gamma(10 \mu \mathrm{g} / \mathrm{ml}$, clone XMG 1.2); and Th17, anti-IL-2R $\alpha$ $(10 \mu \mathrm{g} / \mathrm{ml}$, clone PC61, American Type Culture Collection (ATCC), Manassas, VA, USA), IL-23(10 ng/ml), IL-6 (10 ng/ml), TGF- $\beta 1(1 \mathrm{ng} / \mathrm{ml})$, anti-IFN- $\gamma(10 \mu \mathrm{g} / \mathrm{ml}$, clone XMG 1.2) and anti-lL-4 (10 $\mu \mathrm{g} / \mathrm{ml}$, clone $11 \mathrm{~B} 11)$. At $48 \mathrm{~h}$ after the start of culture, cells were transferred to another plate, which was not coated with anti-CD3 or anti-CD28 Abs, with the original culture media (including polarizing cytokines and anti-cytokine Abs). Cells cultured in the Th1 condition were also supplemented with fresh human IL-2 $(5 \mathrm{U} / \mathrm{ml})$.

Immunofluorescence and TUNEL assay. Cells were adhered to poly-Llysine-coated cover slips, fixed with $2 \%$ paraformaldehyde, permeabilized with $0.1 \%$ Triton X-100 and stained with primary and secondary antibodies. The following primary antibodies were used for immunofluorescence studies: anti-LC3 antibody (purchased from MBL International, Woburn, MA, USA, M152-3), anti-Caspase 3 antibody (purchased from Cell Signaling Technology, Inc., Danvers, MA, USA, 9662), anti-Caspase 8 antibody (purchased from R\&D Systems, Minneapolis, MN, USA, AF1650), anti-p62 antibody (purchased from ARP American Research Products, Inc., Belmont, MA, USA, 03-GP62-C) and anti-Ubiquitin antibody (purchased from Millipore, Billerica, MA, USA, 04-263). The secondary antibodies used were: donkey anti-rabbit Alexa Fluor 488 (Invitrogen, A21206), goat anti-mouse Alexa Fluor 647 (Invitrogen, A21235), goat anti-guinea pig Alexa Fluor 488 (Invitrogen, A11073) and donkey anti-rabbit Alexa Fluor 546 (Invitrogen, A10040). Finally, cells were counterstained with Hoechst 33342 nuclear dye.

For the TUNEL assay, cells were adhered to poly-L-lysine-coated cover slips, fixed with $2 \%$ paraformaldehyde, permeabilized with $0.1 \%$ Triton X-100 and stained with a TUNEL detection kit according to company protocol (Millipore). Finally, cells were counterstained with Hoechst 33342 nuclear dye, courtesy of University of Pittsburgh Center for Biological Imaging. Slides were examined by fluorescence microscopy using an Olympus FluoView 1000 confocal microscope using a $\times 100$ objective (Olympus America Inc., Center Valley, PA, USA). Images are taken consecutively to observe each fluorophore and averaged for three images.

Proliferation assays and flow cytometry. To label cells with CFSE, cells were incubated with $1 \mu \mathrm{M}$ CFSE (Invitrogen) for $10 \mathrm{~min}$ at $37^{\circ} \mathrm{C}$ in PBS and then washed in complete media. Labeled cells were cultured with the indicated stimuli for $72 \mathrm{~h}$ and then analyzed by flow cytometry.

EAE induction. Mice were used for EAE induction by s.c. injection of $150 \mu \mathrm{g}$ $M \mathrm{G}_{35-55}$ (MEVGWYRSPFSRVVHLYRNGK) peptide in IFA plus $500 \mu \mathrm{g}$ of heatinactivated Mycobacterium tuberculosis. One hundred nanograms of pertussis toxin was injected i.v. on days 0 and 2. Mice were monitored for clinical signs of EAE, scored as follows: 0 , normal; 1 , flaccid tail; 2 , hind limb weakness or abnormal gait with poor ability to right from supine; 3 , partial hind limb paralysis; 4 , both hind limbs paralyzed with or without forelimb paralysis and incontinence; and 5, moribund state. The experiments were performed in accordance with the regulation of the Institutional Animal Care and Use Committee of University of Pittsburgh.

CNS cell isolation and $\mathbf{A b}$ staining. To isolate cells from the CNS, mice were deeply anesthetized and perfused intracardially with cold RPMI 1640 medium. Spinal cord cell suspensions were incubated with $1 \mathrm{mg} / \mathrm{ml}$ collagenase II (SigmaAldrich, St. Louis, MO, USA) at $37^{\circ} \mathrm{C}$ for $20 \mathrm{~min}$, then resuspended in $30 \%$ Percoll (GE Healthcare, Piscataway, NJ, USA), which was then layered over $70 \%$ Percoll. Mononuclear cells were separated from the interface after centrifugation at $600 \times g$ for $20 \mathrm{~min}$ and washed in PBS. The total number of cells derived from each animal was determined using a hemacytometer. CNS-derived inflammatory cells were incubated with anti-CD45, anti-CD11b and anti-CD4 Abs for 15 min on ice in Hank's buffer (containing $1 \%$ FCS). Flow cytometric analysis was performed using a FACS flow cytometer (BD Biosciences, San Jose, CA, USA).

Ex vivo analysis of spleen cells, lymph node cells and CNS cells by intracellular cytokine staining. Splenocytes, lymphocytes and CNSinfiltrating leukocytes were cultured with or without $\mathrm{MOG}_{35-55}$ peptide at 50,10 or $0 \mu \mathrm{g} / \mathrm{ml}$ for $18 \mathrm{~h}$. Brefeldin A was added for the last $4 \mathrm{~h}$ at $10 \mu \mathrm{g} / \mathrm{ml}$. Cells were transferred to a V-bottom plate, stained with anti-CD4 in Hank's buffer (containing $1 \%$ FCS) and then fixed with $2 \%$ formaldehyde, which was followed by permeabilization with $0.5 \%$ saponin. The cells were subsequently stained with anti-IL-17 and anti-IFN- $\gamma$ Ab (eBioscience, Inc., San Diego, CA, USA). Flow cytometric analysis was performed using a flow cytometer (BD Biosciences). 
Western blot analysis and immunoprecipitation. Activated T cells were harvested at $48 \mathrm{~h}$, and $\mathrm{CD} 4{ }^{+} \mathrm{T}$ cells were purified using a positive selection method employing biotin-conjugated CD4 antibody (BD Biosciences) and streptavidin-conjugated Microbeads (Miltenyi Biotec, Auburn, CA, USA). Purified CD4 were counted, pelleted and whole-cell lysate was extracted using a cell lysis buffer (containing) to resuspend the cell pellet, followed by $10 \mathrm{~min}$ at $100 \mathrm{C}$. Western blot analysis was performed using 10\% Tris- $\mathrm{HCl}$ Ready Gel (Bio-Rad, Hercules, CA, USA) and run out at $130 \mathrm{~V}$ for $\sim 1 \mathrm{~h}$, followed by transfer to an Immuno-Blot PVDF membrane (Bio-Rad) for $2 \mathrm{~h}$, blocking for $1 \mathrm{~h}$ with $5 \%$ milk, and overnight incubation with primary antibody at $4 \mathrm{C}$. Following a wash $(4 \times, 15$ min wash with $0.1 \%$ Tween in PBS), the secondary antibody is added for $1 \mathrm{~h}$ at room temperature. The membrane is washed again and activated using an Amersham ECL plus western blotting detection system (GE Healthcare). Results were analyzed using a Kodak Image station (Eastman Kodak Company, Rochester, NY, USA). Western blot analysis was performed using following antibodies: Casp3 (9662, Cell Signaling), Casp8 (AF1650, R\&D Systems), p62 (03-GP62-C, ARP American Research Products, Inc), Bim (2819, Cell Signaling) and Bcl2 (554087, BD biosciences). Immunoprecipitation was performed according to published protocol with modification. ${ }^{38}$ Briefly, $5 \times 10^{6}$ T cells were lysed with $0.5 \mathrm{ml}$ lysis buffer $(30 \mathrm{mM}$

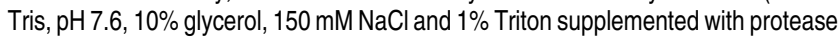
inhibitor cocktail (Hoffmann-La Roche Inc., Nutley, NJ, USA). Immunoprecipitation was performed using $2 \mu \mathrm{g}$ of p62/sqstm1 monoclonal antibody (M01, clone $2 \mathrm{C} 11$, Abnova, Walnut, CA, USA) or control IgG.

\section{Conflict of Interest}

The authors declare no conflict of interest.

Acknowledgements. We thank Drs. Penny Morel and Lisa Borghesi for critical reading, and Ms Christen Shiber for technical assistance. This work is partly supported by National Institutes of Health (NIH) Grant Al063496 to BL and a pilot grant of Cancer and Aging Program (NIH Grant no. P20 CA103730) to BL. JRK is supported by NIH training Grant T32 CA082084. This work is also partly supported by NSFC grants 30528008 (to B Lu and X Zhang). QY and XC are supported by Eleven-Fifth Mega-Scientific Project on 'prevention and treatment of AIDS, viral hepatitis and other infectious diseases' (2008ZX10003-012). GL is supported by a scholarship from China Scholarship council \# 2010692006.

\section{Author Contributions}

JRK and $B L$ designed and performed the experiments and prepared the manuscript. $\mathrm{CL}$ and SJ generated the conditional knockout mice. IGG and QY performed experiments and analyzed results.

1. Cuervo AM. Autophagy: many paths to the same end. Mol Cell Biochem 2004; 263: 55-72.

2. Levine B, Klionsky DJ. Development by self-digestion: molecular mechanisms and biological functions of autophagy. Dev Cell 2004; 6: 463-477.

3. Shintani T, Klionsky DJ. Autophagy in health and disease: a double-edged sword. Science 2004; 306: 990-995.

4. Mizushima N, Klionsky DJ. Protein turnover via autophagy: implications for metabolism. Annu Rev Nutr 2007; 27: 19-40.

5. Klionsky DJ. The molecular machinery of autophagy: unanswered questions. J Cell Sci 2005; 118 (Part 1): 7-18.

6. Suzuki K, Ohsumi Y. Molecular machinery of autophagosome formation in yeast, Saccharomyces cerevisiae. FEBS Lett 2007; 581: 2156-2161.

7. Liang XH, Kleeman LK, Jiang HH, Gordon G, Goldman JE, Berry G et al. Protection against fatal Sindbis virus encephalitis by beclin, a novel Bcl-2-interacting protein. $J$ Virol 1998; 72: 8586-8596.

8. Liang XH, Jackson S, Seaman M, Brown K, Kempkes B, Hibshoosh $\mathrm{H}$ et al. Induction of autophagy and inhibition of tumorigenesis by beclin 1. Nature 1999; 402: $672-676$.

9. Kihara A, Noda T, Ishihara N, Ohsumi Y. Two distinct Vps34 phosphatidylinositol 3-kinase complexes function in autophagy and carboxypeptidase $\mathrm{Y}$ sorting in Saccharomyces cerevisiae. J Cell Biol 2001; 152: 519-530.

10. Zhong Y, Wang QJ, Li X, Yan Y, Backer JM, Chait BT et al. Distinct regulation of autophagic activity by Atg14L and Rubicon associated with Beclin 1-phosphatidylinositol-3kinase complex. Nat Cell Biol 2009; 11: 468-476.
11. Matsunaga K, Saitoh T, Tabata K, Omori H, Satoh T, Kurotori N et al. Two Beclin 1-binding proteins, Atg $14 \mathrm{~L}$ and Rubicon, reciprocally regulate autophagy at different stages. Nat Cell Biol 2009; 11: 385-396.

12. Espert L, Denizot M, Grimaldi M, Robert-Hebmann V, Gay B, Varbanov M et al. Autophagy is involved in T cell death after binding of HIV-1 envelope proteins to CXCR4. J Clin Invest 2006; 116: 2161-2172.

13. Li C, Capan E, Zhao Y, Zhao J, Stolz D, Watkins SC et al. Autophagy is induced in CD4+ $T$ cells and important for the growth factor-withdrawal cell death. J Immunol 2006; 177 5163-5168.

14. Feng CG, Zheng L, Jankovic D, Bafica A, Cannons JL, Watford WT et al. The immunity-related GTPase Irgm1 promotes the expansion of activated CD4+ T cell populations by preventing interferon-gamma-induced cell death. Nat Immunol 2008; 9: 1279-1287.

15. Bell BD, Leverrier S, Weist BM, Newton RH, Arechiga AF, Luhrs KA et al. FADD and caspase-8 control the outcome of autophagic signaling in proliferating T cells. Proc Natl Acad Sci USA 2008; 105: 16677-16682.

16. Pua HH, Dzhagalov I, Chuck M, Mizushima N, He YW. A critical role for the autophagy gene Atg5 in T cell survival and proliferation. J Exp Med 2007; 204: 25-31.

17. Stephenson $\mathrm{LM}$, Miller BC, Ng A, Eisenberg J, Zhao Z, Cadwell $K$ et al. Identification of Atg5-dependent transcriptional changes and increases in mitochondrial mass in Atg5deficient T lymphocytes. Autophagy 2009; 5: 625-635.

18. Pua HH, Guo J, Komatsu M, He YW. Autophagy is essential for mitochondrial clearance in mature T lymphocytes. J Immunol 2009; 182: 4046-4055.

19. Kirkin V, McEwan DG, Novak I, Dikic I. A role for ubiquitin in selective autophagy. Mol Cell 2009; 34: 259-269.

20. Moscat J, Diaz-Meco MT. p62 at the crossroads of autophagy, apoptosis, and cancer. Cell 2009; 137: 1001-1004.

21. Tait SW, Green DR. Caspase-independent cell death: leaving the set without the final cut. Oncogene 2008; 27: 6452-6461.

22. Jaattela M, Tschopp J. Caspase-independent cell death in T lymphocytes. Nat Immunol 2003; 4: 416-423.

23. Degterev A, Huang Z, Boyce M, Li Y, Jagtap P, Mizushima N et al. Chemical inhibitor of nonapoptotic cell death with therapeutic potential for ischemic brain injury. Nat Chem Biol 2005; 1: 112-119.

24. Weant AE, Michalek RD, Khan IU, Holbrook BC, Willingham MC, Grayson JM. Apoptosis regulators Bim and Fas function concurrently to control autoimmunity and CD8+ T cell contraction. Immunity 2008; 28: 218-230.

25. Hutcheson J, Scatizzi JC, Siddiqui AM, Haines 3rd GK, Wu T, Li QZ et al. Combined deficiency of proapoptotic regulators Bim and Fas results in the early onset of systemic autoimmunity. Immunity 2008; 28: 206-217.

26. Hughes PD, Belz GT, Fortner KA, Budd RC, Strasser A, Bouillet P. Apoptosis regulators Fas and Bim cooperate in shutdown of chronic immune responses and prevention of autoimmunity. Immunity 2008; 28: 197-205.

27. Green DR. Fas Bim boom! Immunity 2008; 28: 141-143.

28. Hildeman DA, Zhu Y, Mitchell TC, Bouillet $P$, Strasser A, Kappler $J$ et al. Activated T cell death in vivo mediated by proapoptotic bcl-2 family member bim. Immunity 2002; 16: 759-767.

29. Boatright KM, Renatus M, Scott FL, Sperandio S, Shin H, Pedersen IM et al. A unified model for apical caspase activation. Mol Cell 2003; 11: 529-541.

30. Jin Z, Li Y, Pitti R, Lawrence D, Pham VC, Lill JR et al. Cullin3-based polyubiquitination and p62-dependent aggregation of caspase-8 mediate extrinsic apoptosis signaling. Cell 2009; 137: 721-735.

31. Pankiv S, Clausen TH, Lamark T, Brech A, Bruun JA, Outzen $\mathrm{H}$ et al. p62/SQSTM1 binds directly to Atg8/LC3 to facilitate degradation of ubiquitinated protein aggregates by autophagy. J Biol Chem 2007; 282: 24131-24145.

32. Choi YE, Butterworth M, Malladi S, Duckett CS, Cohen GM, Bratton SB. The E3 ubiquitin ligase CIAP1 binds and ubiquitinates caspase- 3 and -7 via unique mechanisms at distinct steps in their processing. J Biol Chem 2009; 284: 12772-12782.

33. Zhang $X$, Brunner $T$, Carter L, Dutton RW, Rogers $P$, Bradley $L$ et al. Unequal death in $T$ helper cell (Th)1 and Th2 effectors: Th1, but not Th2, effectors undergo rapid Fas/FasLmediated apoptosis. J Exp Med 1997; 185: 1837-1849.

34. Kischkel FC, Hellbardt S, Behrmann I, Germer M, Pawlita M, Krammer PH et al. Cytotoxicity-dependent APO-1 (Fas/CD95)-associated proteins form a death-inducing signaling complex (DISC) with the receptor. Embo J 1995; 14: 5579-5588.

35. Jia W, He YW. Temporal regulation of intracellular organelle homeostasis in T lymphocytes by autophagy. J Immunol 2011; 18: 18.

36. Arsov I, Adebayo A, Kucerova-Levisohn M, Haye J, MacNeil M, Papavasiliou FN et al. A role for autophagic protein Beclin 1 early in lymphocyte development. J Immunol 2011; 186: 2201-2209.

37. Lum JJ, Bauer DE, Kong M, Harris MH, Li C, Lindsten T et al. Growth factor regulation of autophagy and cell survival in the absence of apoptosis. Cell 2005; 120: 237-248.

38. Gal J, Strom AL, Kwinter DM, Kilty R, Zhang J, Shi $P$ et al. Sequestosome 1/p62 links familial ALS mutant SOD1 to LC3 via an ubiquitin-independent mechanism. J Neurochem 2009; 111: 1062-1073. 